\title{
À la mémoire du Dr Claude Balier (1925-2013)
}

In memoriam: Claude Balier M.D. (1925-2013)

${ }^{1}$ Psychologue clinicienne, psychanalyste. Professeur de Psychologie clinique et Psychopathologie à l'Université Paris Descartes, LPCP/EA 4056

37 , rue de Charonne, 75011 Paris, France. fr.neau@free.fr

\author{
Françoise NEAU ${ }^{1}$
}

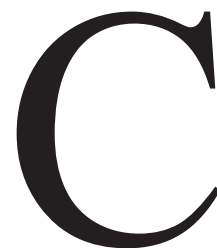

laude Balier, psychiatre, psychanalyste, nous a quittés le 18 mai 2013. Dans ce numéro où sa réflexion est largement convoquée, nous avons souhaité rendre un bref hommage à l'homme et à son œuvre, animés l'un et l'autre par un immense et précieux intérêt pour ce que Claude appelait, simplement, « la qualité de la relation humaine $»$.

Né à Fécamp en 1925, Claude Balier appartient à cette féconde génération de jeunes psychiatres hospitaliers d'après-guerre, qui voulaient transformer profondément la pratique de la psychiatrie en l'humanisant et en la nourrissant de l'approche psychanalytique de la vie psychique.

Interne en psychiatrie dans le service pilote de Paul Sivadon, à Ville-Evrard, puis psychiatre à La Charité sur Loire de 1959 à 1963, il rejoint ses amis Serge Lebovici, René Diatkine et Philippe Paumelle, fondateurs en 1958 de l'Association de Santé Mentale du $13^{\mathrm{e}}$ arrondissement de Paris (ASM 13), tout en entamant à 39 ans une analyse avec Evelyne Kestemberg, à la Société Psychanalytique de Paris, dont il deviendra membre.

Claude Balier participe très activement en 1963 à la création de l'Association de gérontologie et de ses diverses structures de soins, liées à l'ASM 13. Il devient l'un des principaux acteurs du renouvellement de la gérontopsychiatrie en France ; privilégiant avec ses collaborateurs une approche pluridimensionnelle, et psychodynamique, du vieillissement, Claude Balier insiste sur les modifications de l'économie libidinale narcissique que ce vieillissement produit.

À 52 ans, il choisit de quitter Paris. Chef $\mathrm{du}$ service médico-psychologique régional (SMPR) de la maison d'arrêt de Varces près de Grenoble, Claude Balier devient " un psychanalyste en prison »; il le reste jusqu'à la fin de sa carrière, et poursuivra longtemps après ses recherches théoricocliniques, en lançant les fondements d'une importante psycho-criminologie psychanalytique. Auprès des délinquants et des criminels comme auprès des vieillards, la pratique clinique qu'il invente comme psychiatrepsychanalyste, répond à trois préoccupations majeures : aller activement à la rencontre de sujets sans demande, comprendre ces pathologies spécifiques où le recours à l'acte prend souvent face à l'angoisse d'anéantissement chez les plus violents d'entre eux la fonction psychique d'une ultime tentative de sauvegarde, et soigner la personne, au-delà de l'acte commis. Le traitement, que le psychanalyste s'efforce de mener de " regard à regard ", au plus près du transfert dans un " partage affectif " seul à même de rendre possible la rencontre, exige parallèlement l'action thérapeutique de l'ensemble de l'équipe de soins, indispensable pour réduire le clivage massif chez ces sujets. 
Fondateur en 1996 de l'ARTASS (Association pour le Recherche et le Traitement des Auteurs d'Agressions Sexuelles) dont il est resté Président d'honneur, désireux de transmettre son expérience, Claude Balier laisse de nombreux articles et ouvrages, qui éclairent
« l'humanité de l'inhumain », selon le titre de la préface d'André Green à La violence en abyme. Ils sont autant de témoins, à relire et travailler, de l'humanité de Claude, de son esprit d'ouverture et de sa générosité, à partager.

\section{RÉFÉRENCES BIBLIOGRAPHIQUES}

1. Balier C. Le Temps et la vie: les dynamismes du vieillissement (en collaboration avec Jean Guillaumin, Hélène Reboul). Chronique sociale, 1982.

2. Balier C. Psychanalyse des comportements vio lents, Paris, PUF, 1988.
3. Balier C. Psychanalyse des comportements sexuels violents : une pathologie de l'inachèvement, Paris, PUF, 1996.

4. Balier C. La violence en abyme : essai de psychocriminologie, Paris, PUF, 2005

5. Lemaître V., avec la collaboration de Neau F. (rencontre proposée par). La violence de vivre: rencontre avec Claude Balier. Bibliographie raisonnée, Toulouse, Érès, 2007.

\section{Communiqué}

Journée scientifique du Laboratoire Psychologie Clinique, Psychopathologie, Psychanalyse (PCPP) Université Paris Descartes

\section{LA DOULEUR Autour de J.-B. Pontalis}

Samedi 16 Novembre 2013

Faculté de Médecine, 45 rue des Saints Pères, 75006 Paris, France

\section{Organisée par \\ le Pr Catherine Chabert et la revue Carnet Psy}

Jacques André, Catherine Azoulay, Catherine Chabert, Maurice Corcos, Paul Denis, Michèle Emmanuelli, Vincent Estellon, Bernard Golse, Sylvain Missonnier, Françoise Neau, Alexandrine Schniewind Benoît Verdon

\section{Renseignements}

Estelle Georges-Chassot, Carnet Psy, 8 avenue Jean-Baptiste Clément, 92100 Boulogne, France

Tél : 0146047435 - Fax : 0146047400

E-mail : estelle@carnetpsy.com

Site : www.carnerpsy.com 